\title{
Random Spectrometer Motion for Removal of Time Dependent Artifacts in Spectroscopy
}

\author{
P. Gopon, P.E. Sobol, J. Fournelle \\ Dept. of Geoscience, University of Wisconsin, Madison, Wisconsin 53706 USA
}

In Electron Probe Microanalysis (EPMA) spectrometers are used to obtain counts on X-ray peaks, and using the ratio of the counts between a standard and an unknown to determine the composition of the unknown material. An assumption is made that a fixed measurement at the center of an X-ray peak with a Lorentzian shape, represents a set ratio of the entire area the peak. Thus quantitative measurement can typically be made with a single measurement at a fixed energy at the central point of a peak, as opposed to a series of measurements which define the entire peak. Precise determination of peak center position being crucial to EPMA measurements, a series of spectroscopic wavescans are usually conducted before each set of measurements to determine the peak center and shape.

In a typical spectroscopic measurement the value of one dependent parameter is measured vs. variation in an independent parameter. With typical spectroscopic methods the measured signal is acquired while varying the independent parameter in a systematic way, in EPMA for example stepping a narrow band photon detector in a sequence of energy steps from one end of the range of interest to the other monotonically.

One problem that may arise during a spectroscopic analysis is that the specimen or collected signal may vary with time during the data sampling period. For example the specimen composition or chemistry may change as a result of the excitation, or contamination may accumulate on the specimen surface. In the case of long wavescans acquired to determine the center and shape of the low energy $\mathrm{Fe} \mathrm{L} \alpha$ and $\mathrm{Si} \mathrm{K} \alpha$ peaks [1] it was found that the slow accumulation of a carbon contamination layer decreased the intensity of the low energy peaks (Fig. 1) in such a fashion that it distorts the peak shape causing an apparent peak shift in the opposite direction of the wavescan (Fig. 2).

Variation of the independent parameter in a random, pseudo-random or other non-sequential order may reduce or eliminate these artifacts [2]. Standard EPMA software only allows a wavescan to proceed monotonically in one set direction and is susceptible to an apparent peak shift. An algorithm was coded in LabView to allow acquisition of wavescans on the UW Madison electron microprobe in increasing, decreasing and random energy sequences. Figure 1 shows three different wavescan acquisitions of the $\mathrm{Si} \mathrm{K} \alpha$ peak, acquired in monotonically increasing, decreasing and random energy sequence. The two monotonic scans (blue and red) are shifted in the opposite direction in which the scan was acquired, while the random scan (green) falls right in middle of the two other scans and represents the correct shape and position of the peak. The contribution of mechanical backlash to the observed shift is ruled out by the direction of the shift and observations on higher energy peaks [3].

In order to reconstruct the true peak shapes from wavescans acquired with a time dependent distortion (for example due to carbon accumulation) a non-linear fitting algorithm was developed that allows the dependent measurements to be corrected by a lineshape that is a function of the time in the case that the independent parameter is not correlated with time, i.e. in a random ordered scan. This algorithm provides for improved estimation of lineshapes and peak positions from scans suffering from time dependent distortions. 


\section{References}

[1] P Gopon, JF Fournelle and X Llovet, Microsc. Microanal. 18 (Suppl 2) (2012), pp. 1728-1729

[2] P Gopon and PE Sobol, Non-sequential spectral acquisitions to remove time or dose dependent effects, Unpublished report.

[3] RA Fisher, Statistical Methods for Research Workers, $14^{\text {th }}$ Ed., (Oliver \& Boyd, Edinburgh).
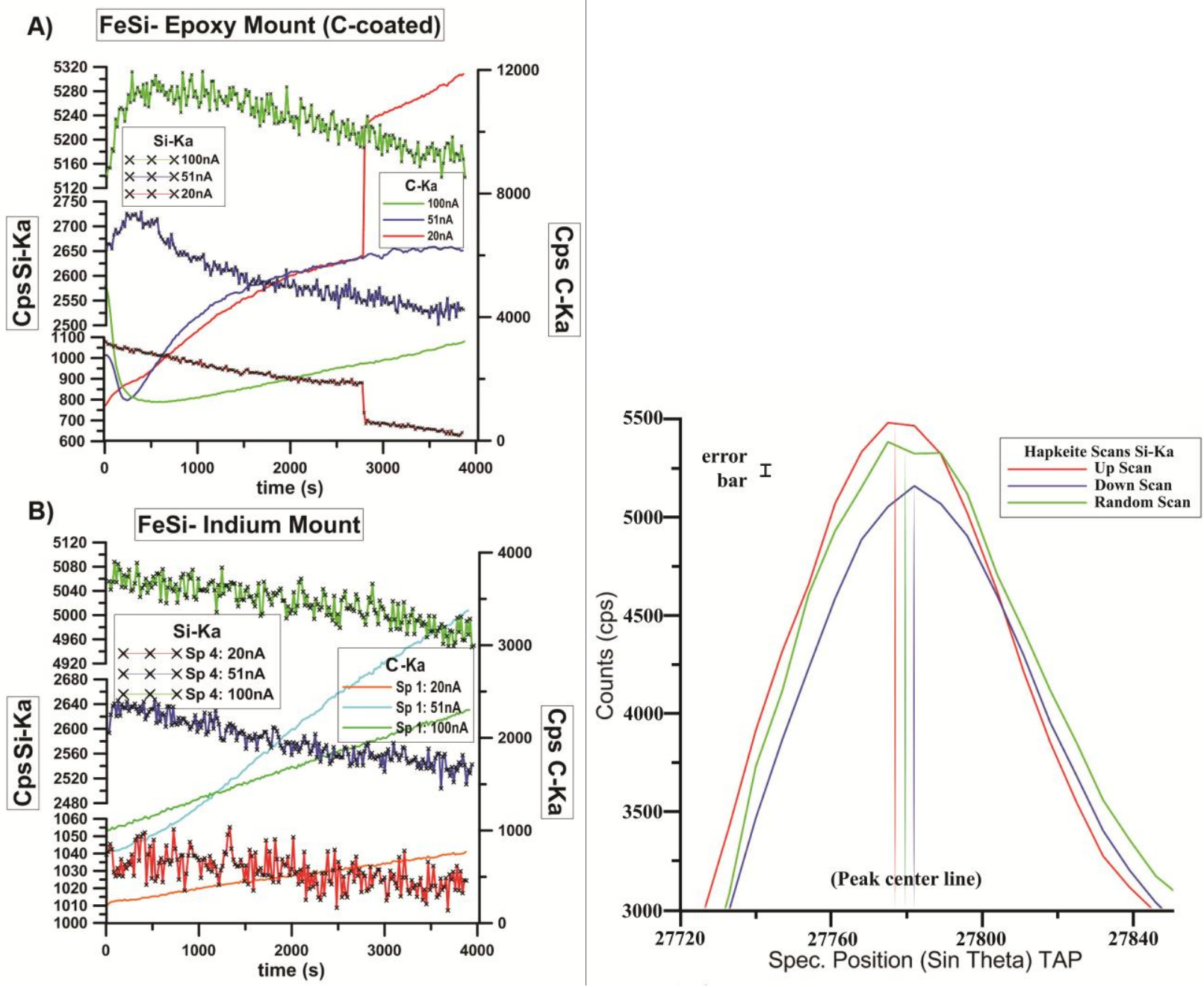

Figure 1. Time dependent variation of $\mathrm{Si} \mathrm{K} \alpha$ Figure 2. Carbon induced apparent peak shifts. counts on FeSi in a carbon coated epoxy mount (a), and an uncoated indium mount (b) for three different currents (all at $5 \mathrm{keV}$ ). The left scale bar (and the data with black Xs) is raw unbackground corrected Si K $\alpha$ cps, while the right scale bar (and the data without the black Xs) is raw unbackground corrected $\mathrm{C} \mathrm{K \alpha}$ cps. The sample colored lines correspond to the same current, so the $\mathrm{Si}$ and $\mathrm{C}$ counts were acquired at the same time. Note that as the $\mathrm{C}$ counts go down the Si counts go up, and vice versa.

Here for long wavescans (1hour) at high current $(100 \mathrm{nA} 5 \mathrm{keV})$ the peak appears to be shifted in the opposite direction of the spectrometer motion used to acquire the wavescan. I.e. when the spectrometer is moved from low to high $\sin \theta$, the peak is shifted to lower $\sin \theta$. The green wavescan is acquired with random spectrometer motion, to remove the time dependency of the carbon contamination spot. Spectrometer motor backlash would account for a peak shift in the direction of the scan, not in the opposite direction as is shown in the figure. 